\title{
High resolution 2D-spectroscopy of granular dynamics
}

\author{
J. Hirzberger ${ }^{1,2}$, M. Koschinsky ${ }^{1}$, F. Kneer ${ }^{1}$, and C. Ritter ${ }^{1}$ \\ 1 Universitäts-Sternwarte, Geismarlandstraße 11, 37083 Göttingen, Germany \\ 2 Institut für Geophysik, Astrophysik und Meteorologie, Universitätsplatz 5, 8010 Graz, Austria
}

Received 16 August 2000 / Accepted 4 December 2000

\begin{abstract}
Spectroscopic data with high spatial resolution are used to study the granular dynamics of the Sun. The observations were obtained with the "Göttingen" two-dimensional (2D) Fabry-Perot interferometer in the Vacuum Tower Telescope at the Observatorio del Teide/Tenerife. Time sequences of spectral scans across the nonmagnetic Fe I 5576 A line were taken from disc center. The 2D spectroscopic images were reconstructed with speckle methods, from which a spatial resolution of $0.4-0.15$ was achieved. A power and coherence analysis of intensity and velocity maps from different photospheric heights has been carried out. The coherence between intensity and velocity fluctuations stays high for structural scales $>0.15$, which underlines the high spatial resolution of the data. Furthermore, the vertical flow field and its time evolution within exploding granules have been analyzed. We find fast downflows in the dark centers of exploding granules with velocities up to $1.2 \mathrm{~km} \mathrm{~s}^{-1}$. Additionally, we estimated the flow velocities of so-called "dark dots". We discuss indications that these structures represent a new type of downflow within the centers of bright granules.
\end{abstract}

Key words. Sun: granulation - Sun: photosphere - techniques: image processing - techniques: spectroscopic

\section{Introduction}

Until a decade ago two principal observational techniques existed for analyzing the fine structure of the solar atmosphere: two-dimensional (2D) imaging on the one side and spectroscopy with slit spectrographs on the other side. In the last years various groups observed so-called twodimensional spectra combining the advantages of both methods (e.g. Johannesson et al. 1992; Salucci et al. 1994; Collados et al. 1996). Together with the improvements of the focal instrumentation sophisticated image reconstruction techniques such as speckle interferometry or phase diversity wavefront sensing have been developed to restore the observed images and thus to overcome the image degradation produced by the Earth's atmosphere (see e.g. Weigelt 1977; von der Lühe 1984; Keller \& von der Lühe 1992; Löfdahl 1996).

The motivation for the development of the new techniques arises from the need for 2D spectroscopic information with high resolution. Only in this way we can improve our knowledge about the dynamics of solar granulation. On the one hand, pure high resolution imaging by, e.g., Roudier \& Muller (1987), Title et al. (1989), Hirzberger et al. $(1997,1999 a)$ have revealed significant differences in the geometrical and brightness structure and the temporal evolution of small and large granules, i.e. large gran-

Send offprint requests to: J. Hirzberger, e-mail: jhirzbe@uni-sw.gwdg.de ules exhibit a rich internal intensity pattern which changes rapidly in time. On the other hand, slit spectrograms (e.g. Kneer et al. 1980; Deubner 1988; Nesis et al. 1988) show a high coherence between velocity and intensity fluctuations in the lower photosphere and a reduction of coherence between these properties in the upper photosphere. Similar results were found from Multichannel Subtractive Double Pass (MSDP) spectrograms as analyzed by Espagnet et al. (1995). Furthermore, observations by Nesis et al. (1992) show that upflow velocities are not symmetrically distributed within the granules, i.e., the fastest upflowing velocities are frequently located in bright edges of large granules. These findings have been confirmed by twodimensional spectroscopic results of Krieg et al. (2000) and are supported by model simulations of Rast (1995). Further references can be found in reviews by Spruit et al. (1990) and Muller (1999).

From these observational studies of solar granulation it has been concluded that the granular dynamics is highly non-linear and that the convective flows are highly turbulent. This view of the granular flows is supported by most of the numerical model simulations (e.g. Cattaneo et al. 1991; Rast 1995; Stein \& Nordlund 1998), although many discrepancies between the different observational studies and between these and numerical model simulations still remain, especially with respect to the turbulence to be expected in the granular flow (Nordlund et al. 1997; Muller 1999; Krieg et al. 2000). The aim of the present paper is to 
demonstrate the advantages of combining two-dimensional spectroscopic observations with efficient image reconstruction techniques. This allows us to measure dynamic properties from images as well as spectroscopic parameters in the solar photosphere down to scales close to the resolution limit of present solar telescopes.

\section{Observations and data analysis}

The observations for the present study were obtained on October 23, 1999, with the "Göttingen" Fabry-Perot interferometer (FPI, for an extensive description see Bendlin et al. 1992; Bendlin \& Volkmer 1995) in the $70 \mathrm{~cm}$ Vacuum Tower Telescope (VTT) at the Observatorio del Teide, Tenerife. The setup was essentially the same as described in Koschinsky et al. (2000), except that the Stokes $V$ polarimeter, which was used by the latter authors, was removed and a glass block with the same optical length as the polarimeter has been inserted. A quiet region at disc center has been observed. The data contain time series of broad band images (50-100 m $\AA$ FWHM, filter centered at $5576 \AA$ ) and simultaneously exposed (synchronized by computer control) narrow band images in the non-magnetic Fe I line at $\lambda=5576 \AA$. The iron line has been scanned at 11 positions (see Fig. 1) with $35.25 \mathrm{~m} \AA$ between adjacent scan positions and 10 images have been taken at each wavelength position. Thus, each scan contains 110 broad band and 110 narrow band images. The exposure time was $20 \mathrm{~ms}$, and the time needed for taking one scan was approximately $30 \mathrm{~s}$ (exposure) and $40 \mathrm{~s}$ (storage), leading to a time interval between two consecutive scans of $70 \mathrm{~s}$. The scale of the images is 0.1 per pixel and the image size is $384 \times 286$ pixel.

The etalon spacing of the narrow band FPI was set to $d=1 \mathrm{~mm}$, its effective finesse is $\mathcal{F} \approx 30$. The resulting $F W H M$ of the Airy function of the FPI in the observed wavelength range is, hence,

$F W H M=\frac{\lambda^{2}}{2 d \mathcal{F}} \approx 52 \mathrm{~m} \AA$

This relatively broad bandwidth leads to a significant broadening of the observed line profile compared to a reference profile. In Fig. 1 the Airy function of the FPI, the reference line profile from the atlas of Brault \& Neckel (1987) taken with the Fourier Transform Spectrometer (FTS), and a convolution of both are overplotted. The advantage of the rather low spectral resolution is that the amount of light, i.e. the number of photons, collected by the narrow band camera is high enough that with an exposure time of only $20 \mathrm{~ms}$ a sufficiently high signal-to-noise ratio can be achieved.

Both broad and narrow band images were carefully corrected for dark offset, flat fields and global image motions. Additionally, the narrow band images were corrected for the transmission curve of the pre-filters ( $F W H M \approx 6 \mathrm{~m} \AA$ ) which has been measured with a halogen lamp as a continuum light source.

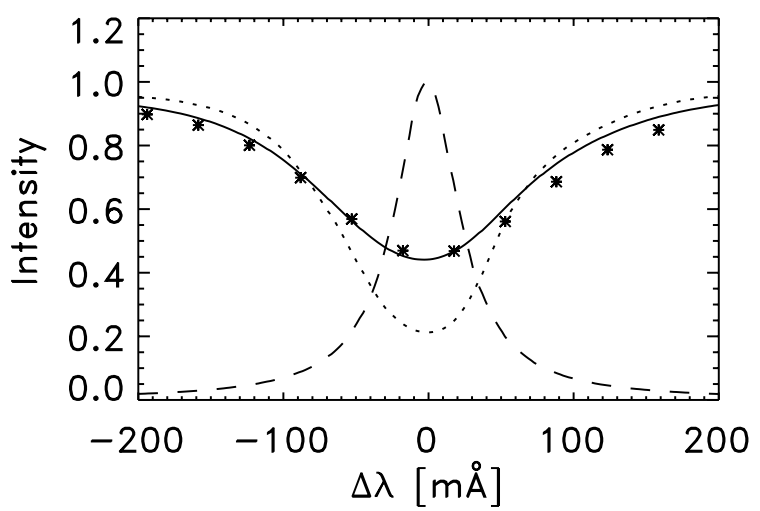

Fig. 1. Spectral resolution of the wavelength scans: mean observed line profile (asterisks); Airy function of the FPI (dashed); reference profile taken from the FTS atlas (dotted); convolution of the Airy function with the reference profile (solid)

In our optical setup the FPIs are mounted in the parallel beam. This prevents flatness errors of the etalons from appearing in the (narrow band) images ("orange peel pattern"). Changes of the finesse across the field of view are negligible. But this setup leads to a wavelength shift of the transmitted light across the field of view. This wavelength shift was calculated by cutting the flat field images into 266 overlapping subfields of $32 \times 32$ pixel and calculating a mean line profile in each of the subfields. The blueshifts of the minima of these 266 profiles were fitted by a paraboloid yielding the blueshifts at each pixel in the images.

The restoration of the broad band images, to correct (to a large extent) for the optical transfer function of the telescope and the Earth's atmosphere, was performed using speckle interferometric techniques (de Boer 1993) based on the speckle masking method (Weigelt 1977) and the spectral ratio method (von der Lühe 1984). The resultant Fried parameters of our observations range from $r_{0}=10.5 \mathrm{~cm}$ to $17 \mathrm{~cm}$, i.e. the images are of excellent quality even without speckle reconstruction.

For the reconstruction of the narrow band images we have assumed that the optical transfer function is the same as in the broad band channel since both were exposed simultaneously and in the same wavelength range. Hence an instantaneous optical transfer function can be estimated from a division (in the Fourier space) of the broad band unrestored images by the speckle reconstructed broad band image (see Krieg et al. 1999). A limitation of this method is the much higher noise level in the narrow band images, compared to that of the corresponding broad band images, which has to be filtered. We use a Wiener filter as given in Brault \& White (1971).

After the flat field correction of the narrow band data the spectral information is lost since at each scan position only relative intensities $I_{\text {rel }, n}^{\prime}(x, y)=I_{n}^{\prime}(x, y) / F_{n}(x, y)$ are remaining ( $n$ denotes the scan position and the prime marks that the images are not yet reconstructed; $F_{n}(x, y)$ are the flat field images). To recover this information a 


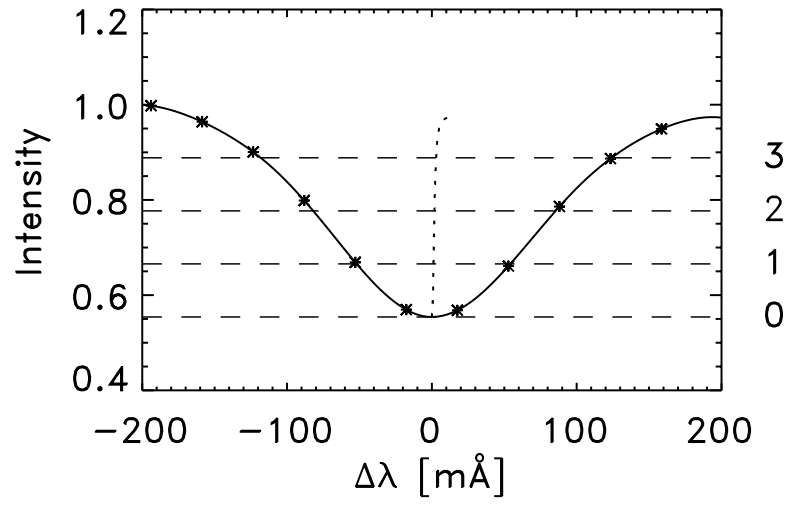

Fig. 2. Levels where intensity and velocity maps have been computed (dashed lines). In Table 1 the corresponding values are listed. The asterisks denote again the averaged observed line profile and the solid line denotes a cubic spline fitted to the observed profile. The dotted curve represents the bisector of the line profile

reference line profile, $I_{\text {ref }}(\lambda)$, was calculated from the flat field images. This was done in two steps: (1) we used the line profiles from the 266 subfields mentioned above and fitted cubic splines to them. (2) Each line profile, i.e. each fitted spline, was shifted until the minimum positions coincided and an average was taken. With this procedure an artificial broadening of the line profile due to the wavelength shift across the field of view could be avoided. Since we know the exact spectral position at each pixel in the images, the relative intensities in the reconstructed images, $I_{\text {rel, }, n}(x, y)$, were multiplied by the corresponding absolute intensity value, $I_{\text {ref }}(\lambda)$, from the reference profile. This retrieves the line intensity at each wavelength position at each pixel.

\section{Intensity and velocity maps}

Now we are able to calculate out of the reconstructed narrow band images line profiles in each pixel of the field of view. Since the Wiener filters cut all the information about structures smaller than 0 ". 3 (the theoretical resolution of the telescope at the observed wavelength range is 0.164$)$ we should not expect a resolution better than 0 .' 3 . Therefore, the line profiles have been averaged over $3 \times 3$ pixel $\left(\widehat{=} 0^{\prime \prime} .3 \times 0^{\prime \prime} .3\right)$ subfields. The smoothed line profiles have been fitted by a cubic spline interpolation and intensity as well as velocity maps have been calculated at four intensity levels in the line profile (see Fig. 2 and Table 1). The velocities have been computed from the bisector of the resulting fitted line profiles. The zero points of the velocity maps have been defined as the mean value in the field of view. We derived from noise a precision of the velocity measurements of $\Delta v_{\text {rms }} \approx \pm 50 \mathrm{~m} / \mathrm{s}$. In Table 1 the rms fluctuations (relative to the mean values at the corresponding wavelength position) of the four intensity maps and the rms fluctuations of the velocity maps from one scan of quiet granulation are given. The intensity fluctuations increase monotonically from the line center up to
Table 1. Parameters of the four intensity and velocity maps for one scan (see also Fig. 2). The intensity levels are given with respect to the local residual intensity $I_{0}$ and the local continuum level $I_{\mathrm{c}}$ and $\Delta \lambda$ gives (approximately) the corresponding wavelength shifts from the line core

\begin{tabular}{llccc}
\hline No. & Intensity Level & $\Delta \lambda[\mathrm{m} \AA]$ & $\Delta I_{\mathrm{rms}}[\%]$ & $\Delta v_{\mathrm{rms}}[\mathrm{m} / \mathrm{s}]$ \\
\hline $0 \ldots I_{0}$ & 0 & 5.54 & 695 \\
$1 \ldots I_{0}+\left(I_{\mathrm{c}}-I_{0}\right) / 4$ & 50 & 5.70 & 715 \\
$2 \ldots I_{0}+\left(I_{\mathrm{c}}-I_{0}\right) / 2$ & 80 & 6.33 & 700 \\
$3 \ldots I_{0}+3 \cdot\left(I_{\mathrm{c}}-I_{0}\right) / 4$ & 125 & 7.06 & 695 \\
$\quad$ broad band & - & 9.93 & - \\
\hline
\end{tabular}

level 3 which is closest to the continuum whereas the velocity fluctuations stay almost constant, i.e. independent on height in the atmosphere. For comparison, the relative rms fluctuations of the corresponding speckle reconstructed broad band image are also shown in Table 1.

Figure 3 shows this broad band image together with the intensity and the velocity map computed at line center. The broad band image appears certainly sharper than the intensity and velocity images from line center. One reason is of course that the former has a better spatial resolution due to the speckle reconstruction method. The second reason is that the latter originate at higher atmospheric layers than the former where the small-scale structures are less pronounced (Komm et al. 1991, see also below, Sect. 4).

Each of the intensity and velocity maps can roughly be associated with a certain height, $z$, in the solar atmosphere which can be estimated by means of so-called temperature response functions, $R F_{\Delta T}(\lambda, z)$, and velocity response functions, $R F_{\mathrm{v}}(\lambda, z)$. (We use the intensity fluctuations as proxies for temperature fluctuations.) The response functions have been derived disturbing the model atmosphere of Holweger \& Müller (1974) in different heights and computing the response of the intensities in a specific spectral line profile (Mein 1971, see also Krieg et al. 1999 and references therein for details of this method).

The temperature response functions for the four levels in the iron line where the intensity maps have been computed are shown in Fig. 4a. The finite spectral resolution has been taken into account, i.e., we have included the Airy function of the FPI (cf. Fig. 1) into the computation of the response functions. For levels 2 and 3 the corresponding response functions are quite narrow and we adopt that most of the contributions to the intensities stem from the continuum level of the atmosphere. For the maps computed at level 0 and 1 the response functions become much broader. Especially the intensity maps calculated in the line center contain an expected contribution from a height of approximately $350 \mathrm{~km}$ above the continuum level and an additional large contribution from the continuum level which is produced by the relatively broad wings of the Airy function of the FPI. Thus we see in the line center intensity maps - as shown in Fig. 3 - still clear granular structures (even though with low contrast) which should not be expected at this height in the atmosphere. 


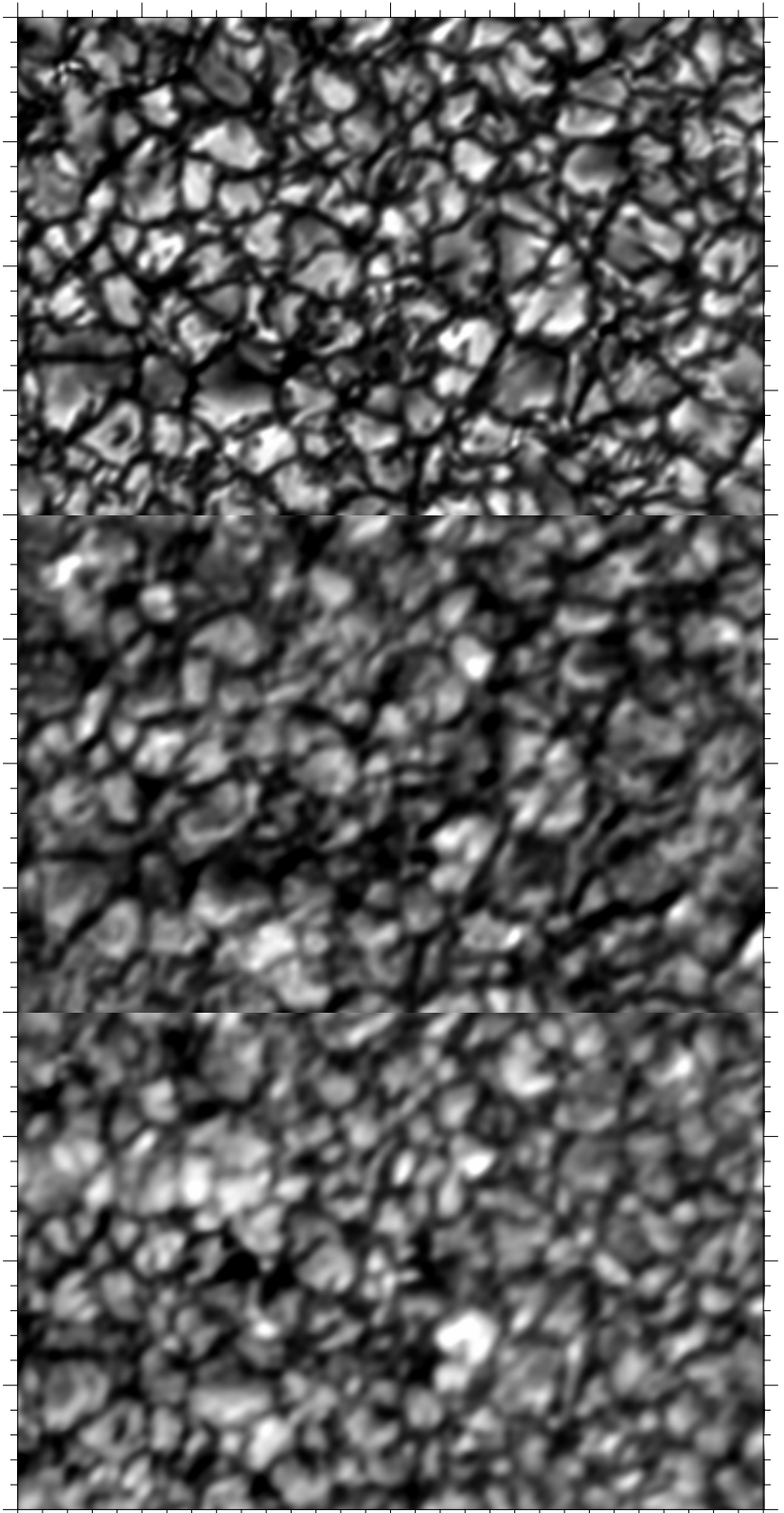

Fig. 3. Example of broad band image (upper panel), intensity map at line center (central panel), and velocity map at line center (lower panel). The tick marks are at distances of $1^{\prime \prime}$

We can disentangle the contributions from many atmospheric heights by using linear combinations of several response functions. In Fig. $4 \mathrm{~b}$ such a combination,

$R F_{\text {tot }}=R F_{0}-0.2 \cdot R F_{1}-0.15 \cdot R F_{3}$,

is plotted. $R F_{0}, R F_{1}, R F_{3}$ denote the temperature response functions calculated at levels 0,1 , and 3 and $R F_{\text {tot }}$ is the resulting response function. In this linear combination the contribution from the continuum level has almost been completely removed and the only contribution originates in a broad region around $z=350 \mathrm{~km}$. The center of gravity of this curve is located at $z=320 \mathrm{~km}$. Applying the same linear combination to the intensity images yields the result shown in Fig. 5. The granular structure has
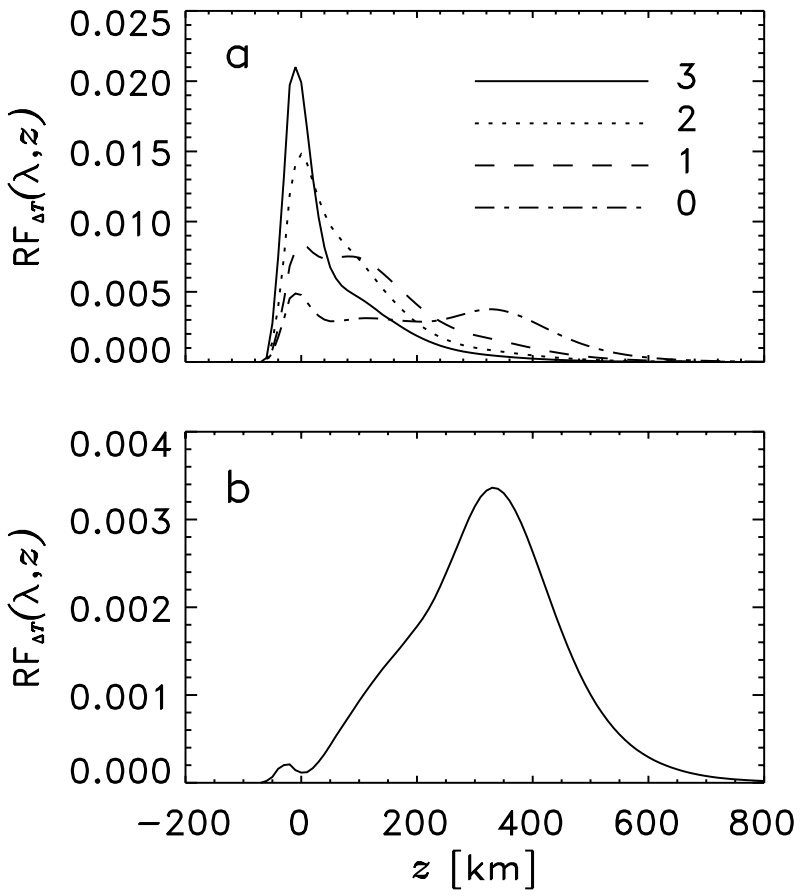

Fig. 4. a) Temperature response functions for the four levels where intensity maps have been computed; b) linear combination of 3 response functions (see text)

disappeared almost completely although some conspicious bright structures (e.g. that one in the upper left corner) are still visible in this image. In the corresponding broad band image (see upper panel in Fig. 3) three small structures which probably are magnetic elements can be detected at this position. It is well known that small-scale magnetic elements expand and become bright in the upper photosphere (see e.g. Stix 1989). Presumably, this large bright structure in Fig. 5 is caused by the magnetic elements which have merged in the middle to upper photosphere. This presumption is corroborated by the inspection of the temporal evolution. The structures are - although changing - present during the whole time span of about $47 \mathrm{~min}$ of the time series.

The velocity response functions for the four levels are shown in Fig. 6. Although they become broader in line center than in regions closer to the continuum their separation is better than that of the temperature response functions. Hence, the resulting velocity maps should contain realistic flow speeds in four different heights in the atmosphere, roughly defined by the center-of-gravity positions of the corresponding response functions, i.e. from $z=250,185,130$, and $100 \mathrm{~km}$.

\section{Power, coherence, and phase spectra}

Helpful diagnostic tools for analyzing and comparing images are power, coherence, and phase spectra. Let $F$ and $G$ be two quantities depending on spatial co-ordinates $x$ and $y$. Then the power spectrum $P\left(k_{x}, k_{y}\right)$ is defined as:

$P\left(k_{x}, k_{y}\right)=\left\langle\tilde{F}\left(k_{x}, k_{y}\right) \cdot \tilde{F}^{*}\left(k_{x}, k_{y}\right)\right\rangle$, 


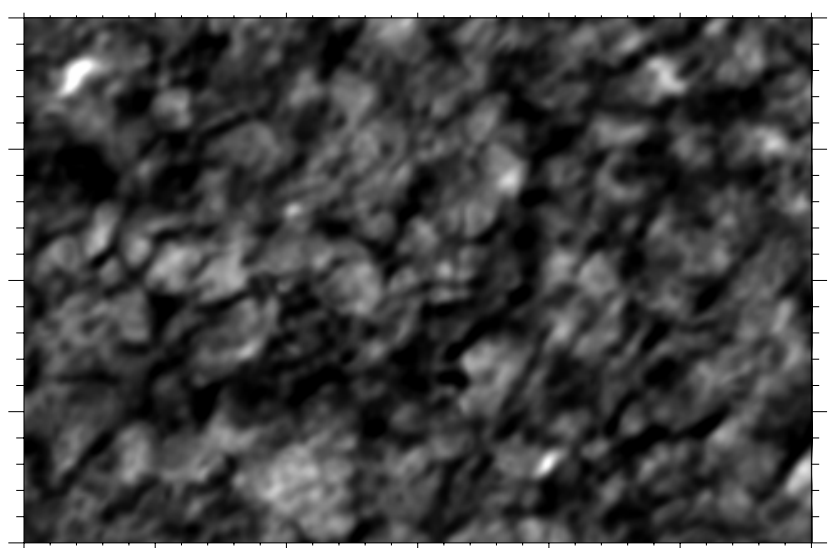

Fig. 5. Linear combination of intensity maps with the prescription of Eq. (2), cf. also the temperature response function in Fig. 4b

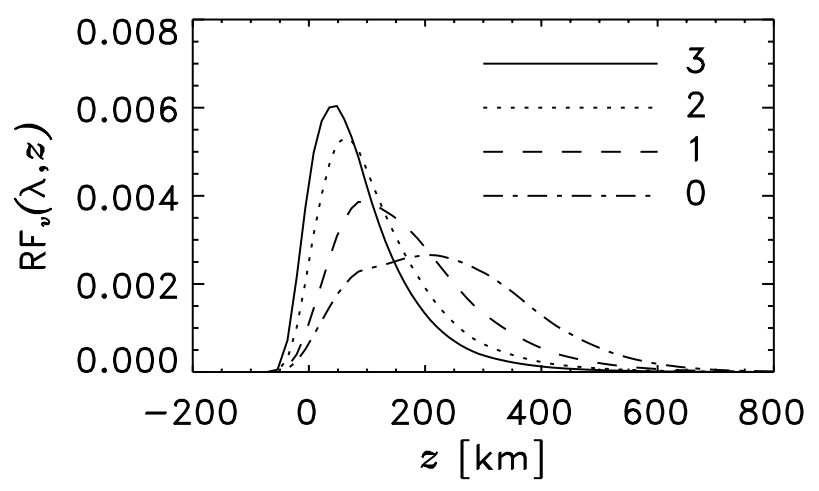

Fig. 6. Velocity response functions for the four levels at which velocity maps have been computed

the phase spectrum is:

$\Delta \Phi\left(k_{x}, k_{y}\right)=\arctan \frac{\Im\left\langle\tilde{F}\left(k_{x}, k_{y}\right) \cdot \tilde{G}^{*}\left(k_{x}, k_{y}\right)\right\rangle}{\Re\left\langle\tilde{F}\left(k_{x}, k_{y}\right) \cdot \tilde{G}^{*}\left(k_{x}, k_{y}\right)\right\rangle}$,

and the coherence spectrum $C\left(k_{x}, k_{y}\right)$ can be written as:

$C\left(k_{x}, k_{y}\right)=\frac{\left|\left\langle\tilde{F}\left(k_{x}, k_{y}\right) \cdot \tilde{G}^{*}\left(k_{x}, k_{y}\right)\right\rangle\right|}{\left\langle\left|\tilde{F}\left(k_{x}, k_{y}\right) \cdot \tilde{G}^{*}\left(k_{x}, k_{y}\right)\right|\right\rangle}$,

depending on wavenumbers $k_{x}$ and $k_{y}$. The $\sim$ denotes Fourier transformed quantities and the asterisk the complex conjugate. Brackets stand for averages over a suitable region, e.g. azimuthal averages in the $k_{x}-k_{y}$-plane as in the following.

Power spectra of the intensity and velocity fluctuations are depicted in Fig. 7. For large wavenumbers $\left(k=\left(k_{x}^{2}+k_{y}^{2}\right)^{1 / 2}>3 \mathrm{Mm}^{-1}\right)$ power increases from the line center to continuum, i.e. the intensity as well as the velocity fluctuations decrease with increasing photospheric height. For wavenumbers below $k=3 \mathrm{Mm}^{-1}$ this trend disappears for the intensity fluctuations and becomes even opposite for the velocity fluctuations, i.e. they are larger in
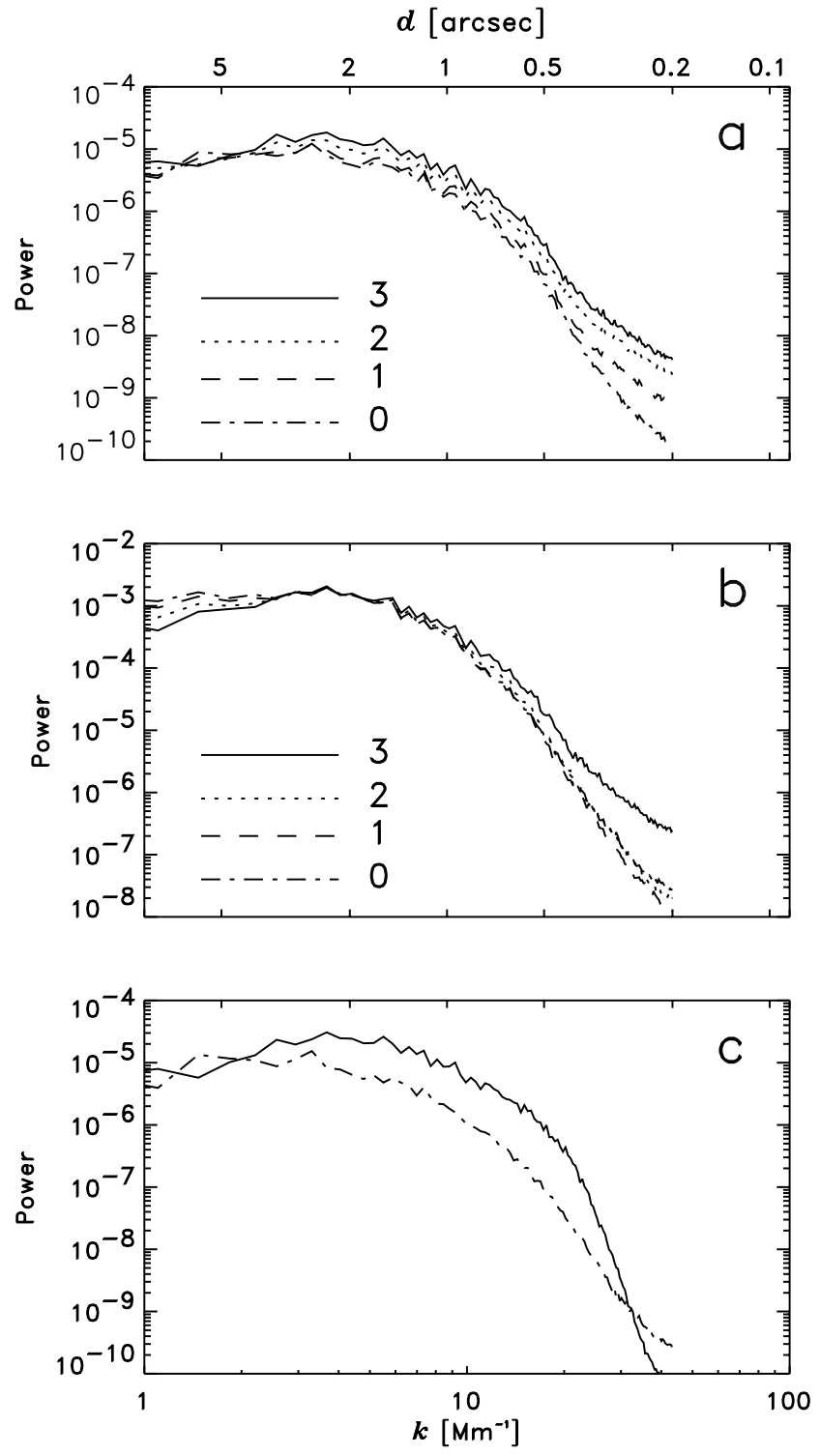

Fig. 7. Power spectra from the four levels in the line profile; a) intensity fluctuations; b) velocity fluctuations; c) broad band image (solid) and the linear combination of intensity maps from Fig. 5 (dash-dotted)

the line center than close to the continuum. Hence, the areas under the curves remain almost constant and the rms fluctuations (the square-root of the integral of the power spectrum) do not change in the different velocity maps (see Table 1). This result coincides with earlier findings of Muller (1985) and Collados et al. (1996). The fluctuations in this range as well as at very low wavenumbers might be attributed, in part, to solar p-modes which are generally more dominant in the velocity than in the intensity fluctuations. Although evanescent in the photosphere, their amplitude increases with height in the atmosphere, which explains the increase of power with height at large scales (e.g. Deubner 1974).

Coherence and phase spectra between the broad band image (displayed in Fig. 3) and the intensity 

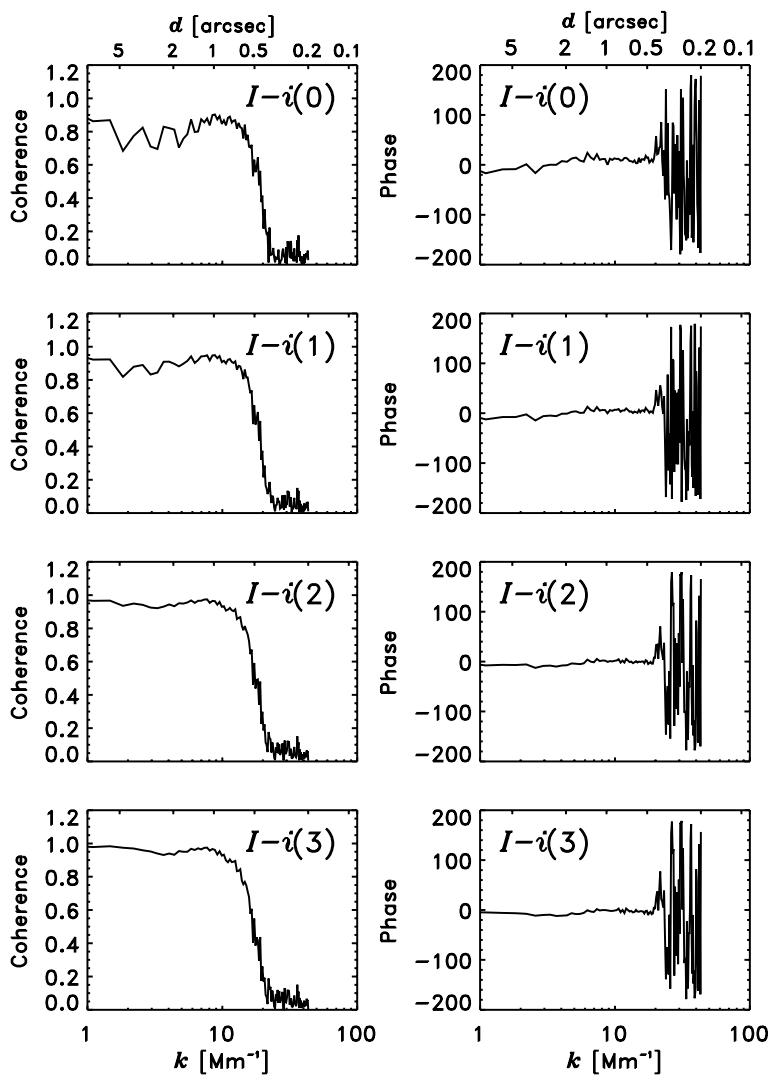
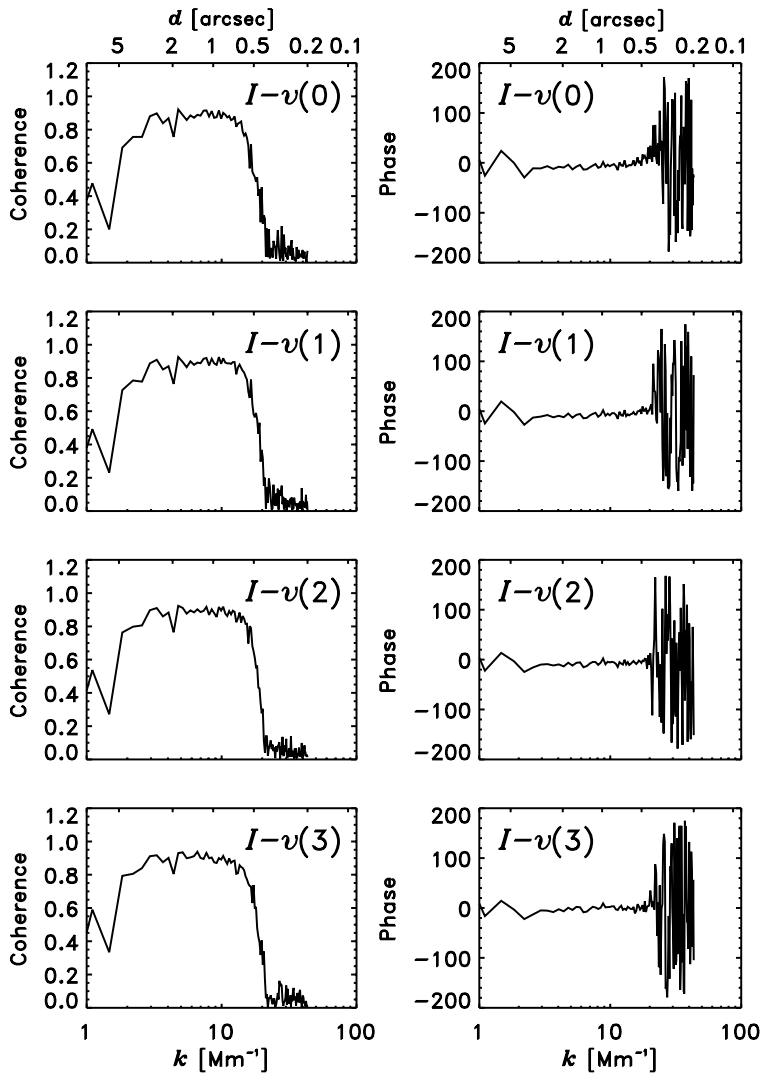

Fig. 8. Coherence and phase spectra between the broad band image, $I$, shown in Fig. 3 and the intensity, $i$, and velocity fluctuations, $v$, from level 0 (top row) to level 3 (bottom row)

fluctuations ( $I$ - $i$-coherences) and the velocity fluctuations ( $I$ - $v$-coherences) calculated at the four intensity levels shown in Fig. 2 and in Table 1 are plotted in Fig. 8. The coherence and the phase between the broad band image and the linear combination of the intensity map from Fig. 5 are shown in Fig. 9. Close to the continuum (levels 2 and 3) the $I$ - $i$-coherences do not deviate from unity in the range $k<10 \mathrm{Mm}^{-1}$, i.e. for structures larger than 0. . 87 . In the same range the phase remains close to zero, i.e. the broad band image and the intensity maps are almost identical at low wavenumbers. Higher up in the photosphere (at the levels 0 and 1 ) the coherence is not so good which means that the granular structure disappears. This is especially valid for the linear combination of intensity maps which should represent information from the heights where the line center is formed.

At large wavenumbers the $I$ - $i$-coherences (at levels 2 and 3) start to decrease and cross the 0.5 -level at $k \approx$ $20 \mathrm{Mm}^{-1}$ (㐱 $\left.0^{\prime \prime} 43\right)$. Also the phases become noisy and begin to oscillate about zero. For $k>20 \mathrm{Mm}^{-1}$ these oscillations cover the entire range between $-180^{\circ}$ and $+180^{\circ}$ and the coherence spectra are significantly below 0.5 . Since the broad band image and the intensity map at level 3 should be - apart from spatial resolution - nearly identical, 0 ". 43 can be taken as the resolution limit of the narrow band data, i.e. before noise dominates the signal. Of course, this value is given by the Wiener filters which start to drop from 1 to 0 at this wavenumber.
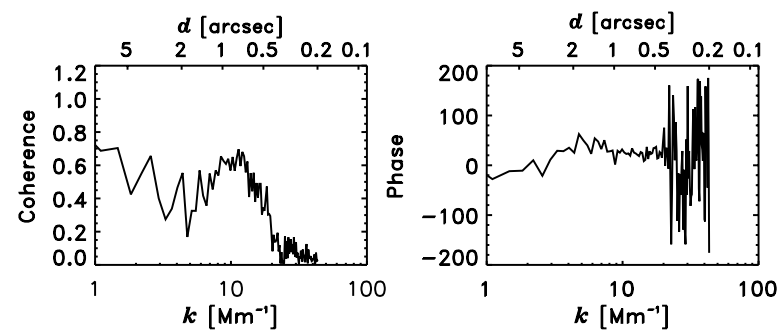

Fig. 9. Coherence spectra (left) and phase spectra (right) between the broad band image and the linear combination of intensity maps shown in Fig. 5

The $I$ - $v$-coherences look somewhat different. In all heights a decrease of coherence towards very low wavenumbers $\left(k<3 \mathrm{Mm}^{-1}\right)$ can be noticed. In this range also the phases deviate significantly from zero. Both results indicate the presence of p-modes. It is well known from theoretical studies that the $I-v$ phase shift of lowfrequency evanescent adiabatic waves is 90 degrees (cf. Noyes \& Leighton 1963). In the range $3<k<12 \mathrm{Mm}^{-1}$ (between 2.9 and 0.77 ) the coherences are in all heights close to 1 . For larger wavenumbers the coherences drop quite fast and cross the 0.5 level again at $k \approx 20 \mathrm{Mm}^{-1}$.

Less noisy and statistically highly significant coherence spectra are shown in Fig. 10. Plotted are $I$ - $i$-coherences and $I$ - $v$-coherences averaged from 54 scans of quiet granulation. The behaviour of these averaged curves reflects 

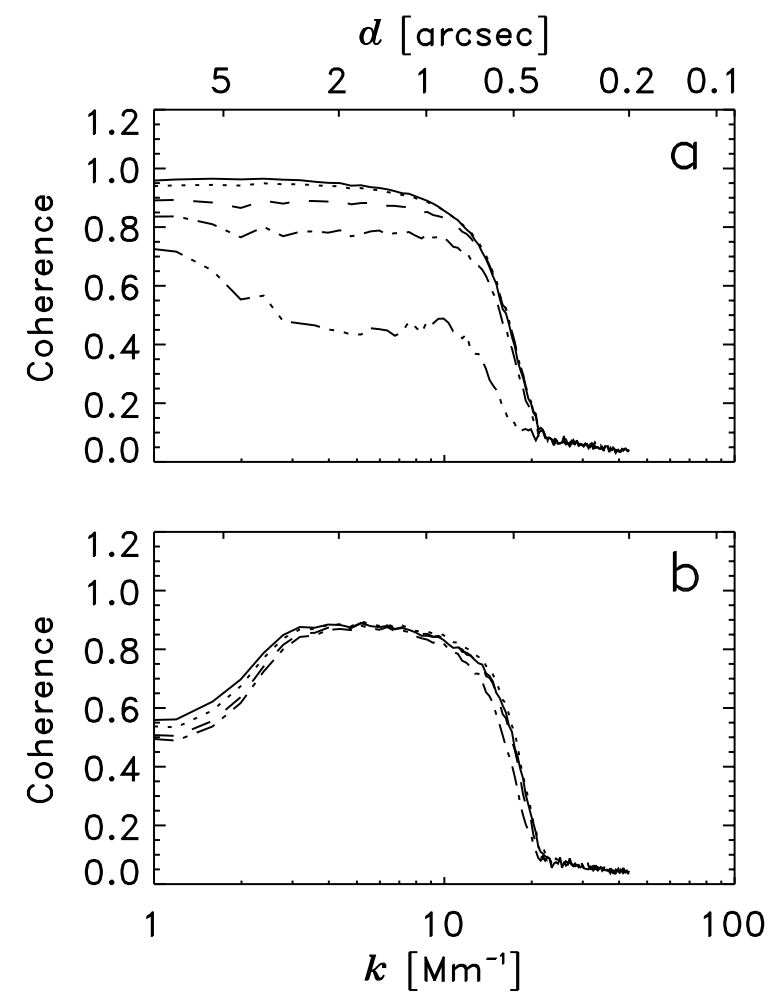

Fig. 10. Averaged $I$-i-coherences a) and $I$ - $v$-coherences b). The line styles are the same as in Figs. 4, 6, and 7. In panel a) the dash-dot-dot-dot curve denotes the averaged coherence between the broad band image and the linear combination of intensity maps

quite well the trend from the single scan described above. Notice that the coherence is high for structures larger than 0. . 5 .

The results from our coherence analysis show that the intensity fluctuations originating from the granular convection diminish much lower in the photosphere than the corresponding velocity fluctuations. This result agrees well with earlier measurements by, e.g., Deubner (1988), Nesis et al. (1988), Salucci et al. (1994), and Espagnet et al. (1995). Additionally, in these studies an inversion of the intensity fluctuations in the high photosphere, above $250 \mathrm{~km}$, was found. Similar results were also predicted from numerical simulations by Steffen et al. (1989). We do not find this inversion, although the linear combination of the temperature response functions in Fig. $4 \mathrm{~b}$ comprises the high photospheric layers. The coherence between the intensity fluctuations in these layers and the broad band granular intensities is low, though (cf. Figs. 9 and 10a).

Concerning the drop of the coherence spectra our results disagree significantly from former studies. We find large values of $I-v$-coherences down to structural scales of 0.5 . This is about a factor of two smaller than those found in Salucci et al. (1994) or Espagnet et al. (1995). We attribute this difference to the very high spatial resolution of our data.

\section{Evolution of exploding granules}

Our knowledge about the flow fields of individual granular structures is only modest. There are two reasons for this: (1) One-dimensional spectrograms from slit spectrographs, even when they exhibit high definition, lack the information of the second horizontal co-ordinate. (2) The spatial resolution of $2 \mathrm{D}$ spectra was moderate, hitherto. The very high spatial resolution of our speckle reconstructed data, hence, makes them suitable for an analysis of the flow fields of individual granules.

A very conspicuous type of granules are the so-called exploding granules. These structures - discovered by Carlier et al. (1968) - can be described by an increase of their size in time and by the development of a dark center. Ever since they have been discovered, their dark centers have been explained by a reverse of the flow direction, i.e. by the formation of a fast downflow. A proof of this explanation is still missing because the size of these dark centers is usually below the spatial resolution of the available spectroscopic data.

Since we have observed time series of granulation scans we are able to follow several of such exploding granules in time. The evolution of the intensity structure and the vertical flow field of a typical exploding granule is shown in Fig. 11. The formation of the downflow in the center of the granule can be seen quite well. Before the granule splits, i.e. in the fourth row of Fig. 11, the maximum velocity of the downflow in the center of the granule (at level 3) is $710 \mathrm{~m} / \mathrm{s}$. Figure 11 demonstrates again the high coherence between the granular intensity pattern (left column) and the granular velocities at various atmospheric heights (middle and right column).

Figure 12 depicts the variation of the center intensities and velocities, averaged over 30 events of exploding granules. The broad band intensities drecrease from a value of about 1.13 (in units of the mean photospheric intensity) to 0.88 within about $8 \mathrm{~min}$. After that the intensity stays almost constant, i.e, the granules have been split and the intergranular intensity is achieved. The central flow velocity decreases almost in parallel from about $950 \mathrm{~m} / \mathrm{s}$ (upward) to approximately $-700 \mathrm{~m} / \mathrm{s}$ (downward).

Before the averaging of the 30 evolutionary histories could be carried out it was necessary to shift them in time above each other. This has been done by defining the starting point of the explosion when the first darkening in the center of the granule was discernible. Hence, the time span of $8 \mathrm{~min}$, when the plateaus of the two curves in Fig. 12 are reached, defines a mean value of the duration of an explosion instead of a lifetime of an exploding granule which can be more than twice as long (see e.g. Hirzberger et al. 1999a).

The result in Fig. 12 demonstrates clearly that the development of the dark center in exploding granules is intimately related to the formation of a downflow. The velocities in this figure are calculated in level 3, i.e. close to the continuum. When tracking the evolution of the velocity in the other levels the results are essentially the same, so they 

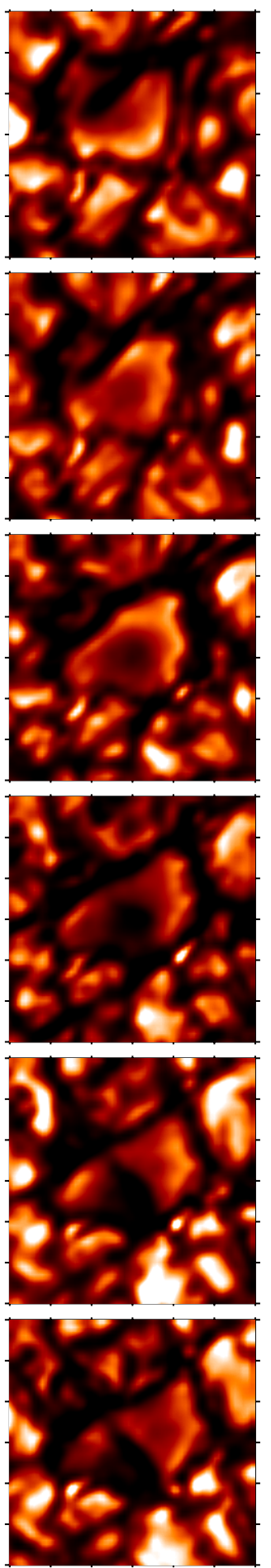
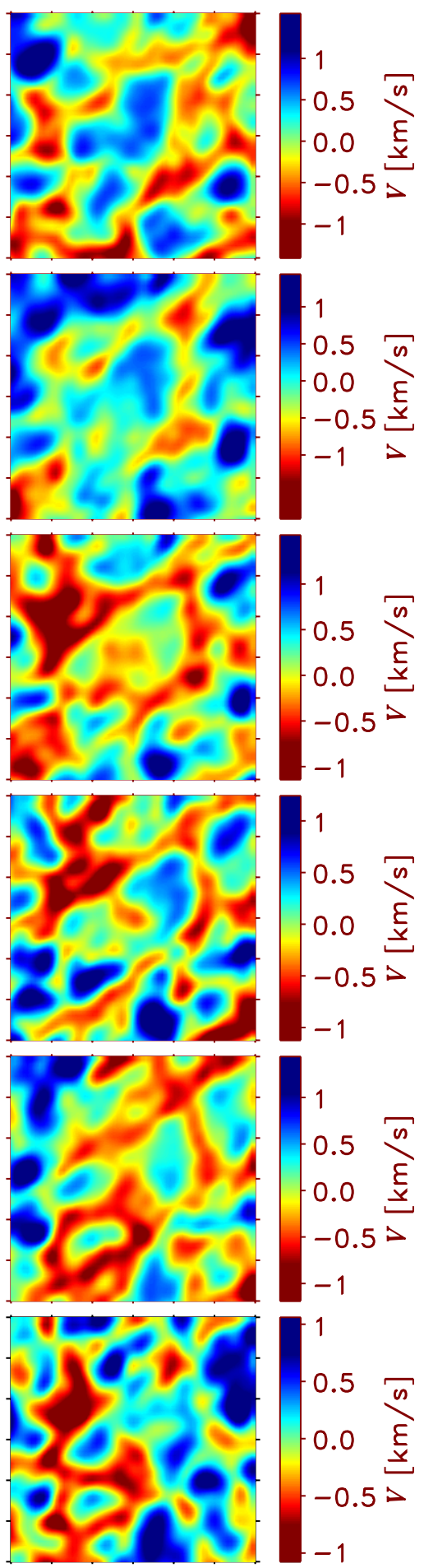
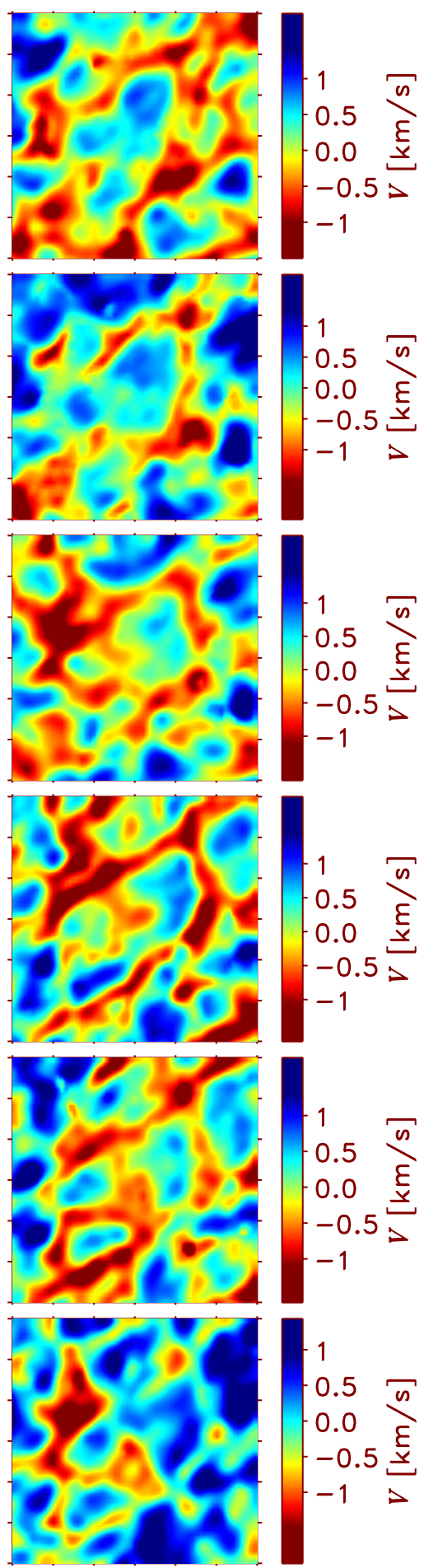

Fig. 11. Time evolution of a typical exploding granule. Left column: broad band images; middle column: flow maps from the line center; right column flow maps from level 3. Time runs from top to bottom in intervals of 70 s. Negative values denote downflows. The distance between the tickmarks is $1^{\prime \prime}$

are not shown in Fig. 12 (but see Fig. 11). We do not find a time delay of the starting point of the velocity decrease in the different heights. At first sight, this result is surprising because numerical models (e.g. Rast 1995; Stein \& Nordlund 1998) explain the explosion of granules by a plume moving from the upper photosphere downwards into deeper layers. If we assume that the vertical distance between the layers where the flow maps at level 0 and 3 are formed is $150 \mathrm{~km}$ and the distance between two spectral scans during observation is $70 \mathrm{~s}$ the flow speed must be higher than $2.14 \mathrm{~km} \mathrm{~s}^{-1}$ to ensure that such a temporal shift of the starting points of the explosion would escape detection. But such a high value is not seen in the measured velocities in Fig. 12. Therefore, the vertical extent of 

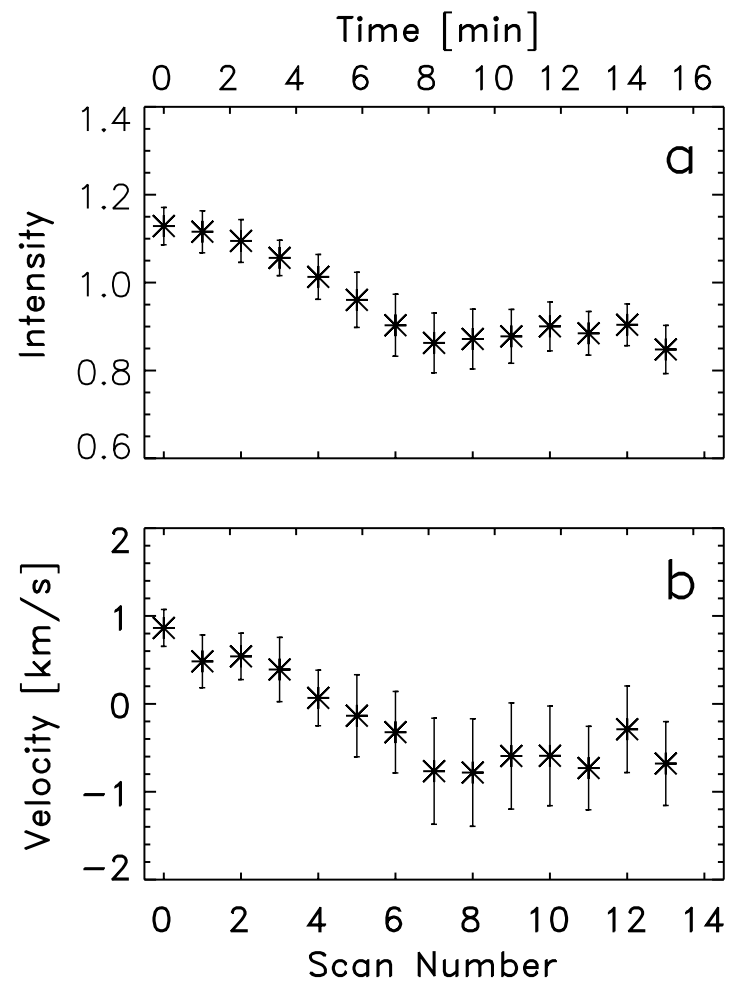

Fig. 12. Averaged time evolution of 30 exploding granules. a) broad band intensities of the granule centers; b) vertical velocities (computed at level 3) of the granule centers. The error bars represent the standard deviations from averaging the 30 individual evolutions

the downflowing plume must cover a large fraction of the distance between the uppermost and the lowermost layer in our study. But since the diameter of the plume is typically in the range of several tenths of an arcsecond (see Fig. 11) a first order estimate would be that its vertical extent is of the same size.

\section{Dark dots}

Exploding granules are not the only granular structures which develop dark centers. Some of these dark centers are rather small and do not expand. These so-called "dark dots" have been first described by Kitai \& Kawaguchi (1979). A systematic analysis of this phenomenon has been given by Hirzberger et al. (1999b). They have shown that dark granular centers are quite frequent and can have sizes from $0{ }^{\prime \prime} 3$, or even smaller, up to more than $1^{\prime \prime}$, i.e. the size of the centers of the largest exploding granules. The nature of these dark dots is still unknown. Hirzberger et al. (1999b) suggest that they are another type of downflow within granules. Gas which is advected into these downflows always has a non-zero angular momentum with respect to the center of the dark dots since the granules which supply a downflow are randomly positioned around it. Consequently, due to conservation of angular momentum, the downflowing gas must rotate around the dark dot's axis and the angular velocity must be proportional to the inverse of the diameter of the downflow. Hence, they possibly look like draining bathwater or tornados. The existence of such small rotating downflows has been also predicted from numerical simulations by Nordlund (1985).

As demonstrated in Sect. 4 the resolution of our data is 0.5 or better. Therefore, we should be able to confirm the idea of downflow. In Fig. 13 the flow velocities within five dark granular centers are compared. The uppermost example shows a large fully developed exploding granule. Its dark center has a "Y"-like shape with a size of 2 ". The velocity profile shows clearly a fast downflow throughout the entire dark region and the bisector from the center of the dark region indicates a flow velocity of more than $1.2 \mathrm{~km} \mathrm{~s}^{-1}$ in all heights. The sharp kink in the upper part of the bisector is probably not real. It results from uncertainties in the reproduction of the line profile close to the continuum level when the Doppler shift is very large because the scanned wavelength region is only approximately $\pm 200 \mathrm{~m} \AA$ (see Figs. 1 and 2).

The second example also shows an exploding granule. But it is at the beginning of its expansion phase, i.e. it is not yet fully developed. The size of the dark center is approximately 0 !' 8 . The velocity profile exhibits a downflow of only about $0.6 \mathrm{~km} \mathrm{~s}^{-1}$ and the bisector is significantly inclined. This inclination means that the flow velocity decreases from about $0.8 \mathrm{~km} \mathrm{~s}^{-1}$ at the line center to almost zero at the continuum level. Hence, a steep vertical velocity gradient must exist here in the photosphere. Possibly this is a hint that indeed the explosion of granules is driven by a dense plume which moves downwards through the photosphere.

In the third row of Fig. 13 a dark dot with a diameter of approximately 0.5 is displayed. The velocity profile as well as the bisector still show negative velocities of approximately $0.4 \mathrm{~km} \mathrm{~s}^{-1}$. The vertical velocity gradient is rather small throughout the entire vertical range.

The fourth and the fifth example in Fig. 13 show dark dots with sizes below 0.5 . The corresponding velocities are slightly negative in the fourth example and slightly positive in the last example. But this does not necessarily mean that the flow direction is indeed upwards. Since we are close to the resolution limit of the data we must consider a large fraction of scattered light from the outer bright part of the granules in which the dark dots are embedded. The Doppler shifts in this scattered light of course indicate positive velocities since most of it comes from bright granular regions.

Although we are not able to measure negative velocities in all dark dots smaller than 0.5 , the existence of downflows is highly probable. In all examples shown in Fig. 13 the velocity profiles exhibit a minimum in the center of the dark dots. The absolute values of the flow velocity in these minima are anticorrelated with the size of the dark dots. This holds also for both the continuum and the line center intensities. We recall from the coherence analysis in Sect. 4 that the critical size in our flow map where the signal-to-noise level falls below unity is around 0.5 . Thus, it is not surprising that for structures smaller 

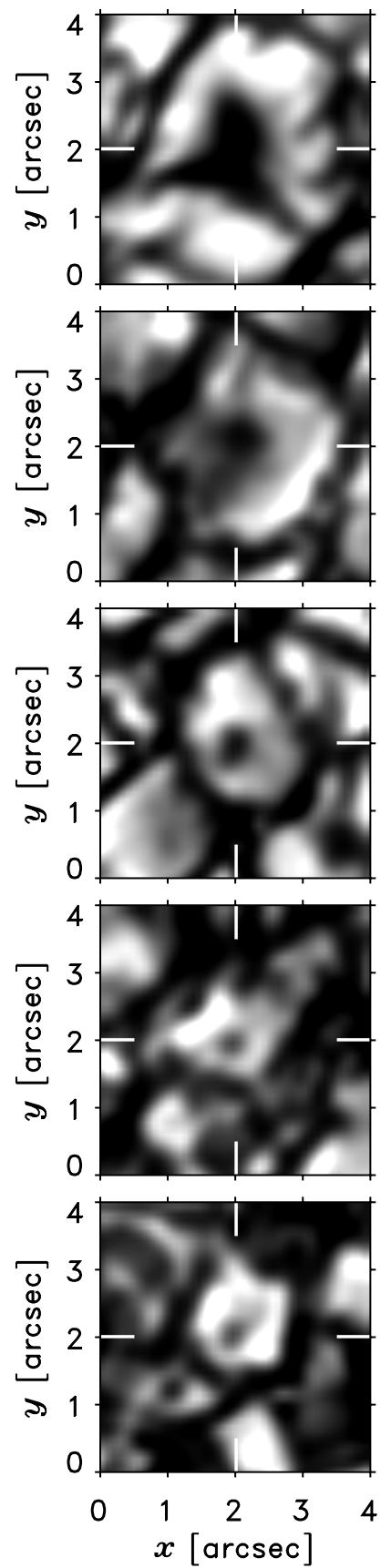
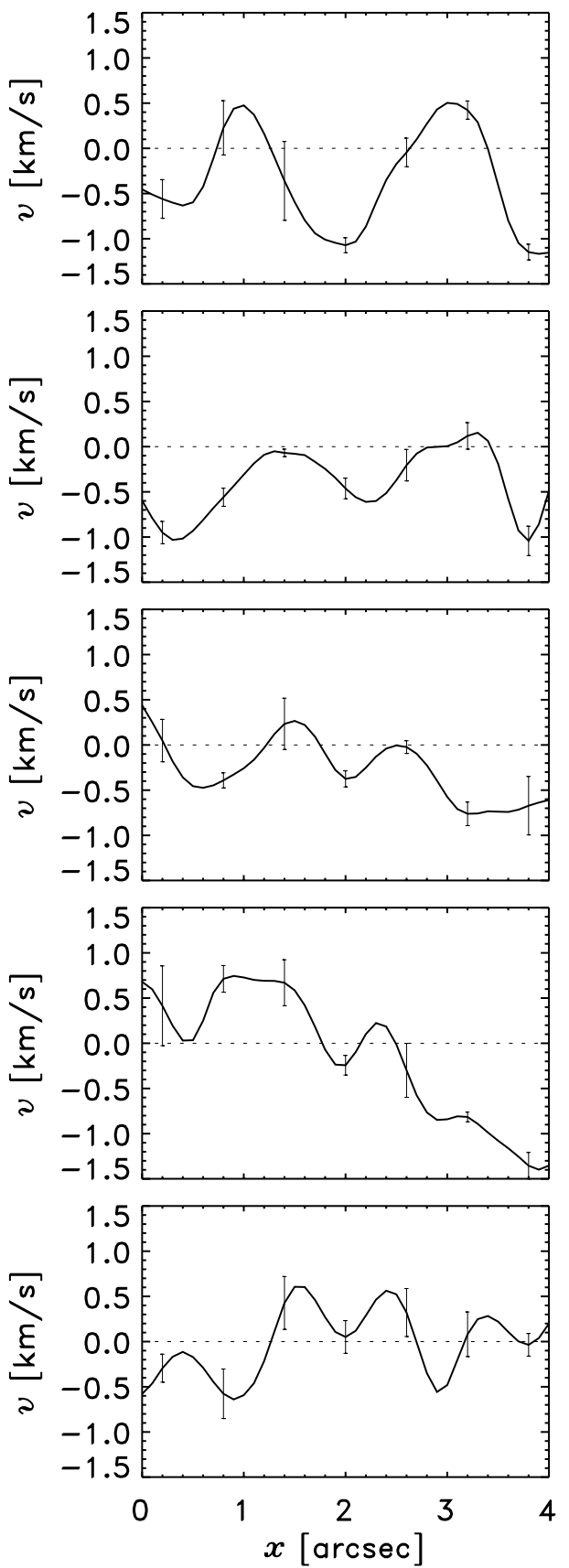
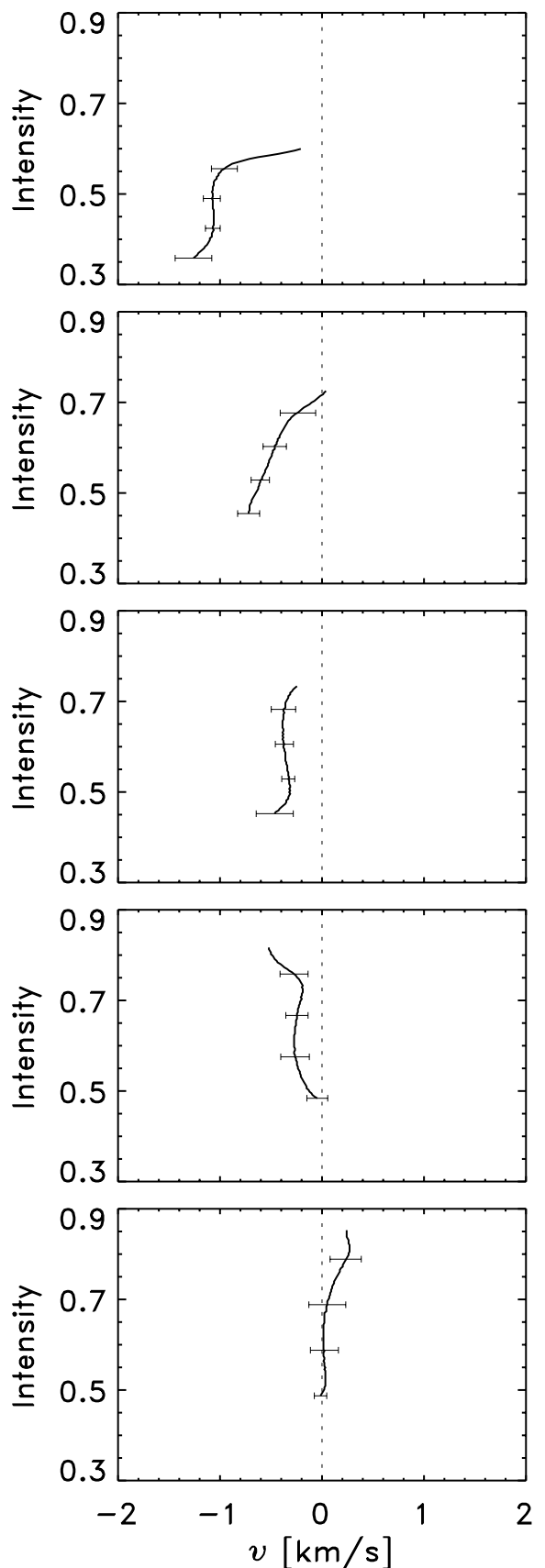

Fig. 13. Dark granular centers of different sizes. Left column: broad band image; middle column: velocity profile (level 2) of the horizontal line at $y=2^{\prime \prime}$; right column: bisector at $x=2^{\prime \prime}, y=2^{\prime \prime}$. Intensities are given in units of the mean photospheric intensity in the continuum. The error bars denote standard deviations when averaging in $3 \times 3$ pixel fields around the position where the velocity is measured

than this value the scattered light dominates the signal. Moreover, we can find in our data many examples with opposite behaviour (e.g. the left part of the exploding granule shown in the second row of Fig. 13): small structures of intermediate brightness which are located within broad intergranular regions show a maximum of the flow velocity but the value of the maximum is below zero presumably because the signal is dominated by scattered light from the surrounding intergranular lanes.

\section{Conclusion}

The combination of two-dimensional spectroscopy with image reconstruction techniques based on speckle interferometry is a powerful tool to gain new insights in the dynamics of solar granulation. We are able to resolve the vertical flow field and the intensity fluctuations for structures with sizes of $0.4-0 . .5$ in different heights of the solar photosphere. For structures with sizes above one 
arcsecond our results agree well with those of earlier one- or two-dimensional spectroscopic measurements. The intensity fluctuations vanish rapidly with photospheric height whereas the flow velocities stay almost constant up to $250 \mathrm{~km}$ which approximately represents the uppermost probed photospheric level.

Additionally, we have demonstrated that the $I-v$ coherences ( $I$ for the broad band image, $v$ for velocity) remain at high values down to structural scales of 0.5 . The $I$ - $i$-coherences ( $i$ for intensity maps in a spectral line) decrease substantially with height in the atmosphere where $i$ is observed. This cannot be seen in spectrograms with lower spatial resolution. The drop of the $I$ - $v$-coherences for structures with sizes below $1^{\prime \prime}$ was sometimes attributed to turbulent motions, i.e. to the turbulent cascade of fragmenting granules. From our results it has to be concluded that motions are not "turbulent" in the range of $0.5-1$ ". 0 in the sense that they do not reduce the correlation between intensities and velocities.

Furthermore, we have shown that the development of dark centers of granules is accompanied by a reversal of the flow direction, i.e. dark centers of granules represent fast downflows. This is especially valid for exploding granules for which this has been suggested already several decades ago. The velocities within the downflows reach values of more than $1.2 \mathrm{~km} \mathrm{~s}^{-1}$ and are more or less constant throughout the entire probed height of the photosphere. Only at the onset of the explosion a steep vertical velocity gradient could be detected. This would confirm the prediction of several numerical simulations (e.g. Rast 1995; Stein \& Nordlund 1998) that the explosion of granules is caused by the downward motion of a plume of dense matter. However, the time resolution of our data is not sufficiently high for a detailed analysis of the dynamic processes that cause the explosion of granules.

Downflows have also been detected in so-called dark dots, i.e. in dark centers which do not expand. These phenomena are sometimes very tiny structures with sizes below 0.5 . For theoretical reasons such small structures have to rotate because the inflowing matter has a nonzero angular momentum. The theoretical reasoning is still unproven because we have no information about the horizontal flow velocities. Future observations at various heliocentric angles off disc center can hopefully clarify this point.

Acknowledgements. The authors are grateful to an anonymous referee for careful reading and for constructive criticism and help. Financial support by the Austrian Fonds zur Förderung der wissenschaftlichen Forschung (ErwinSchrödinger-Stipendium J-1802 PHY) and by the Deutsche Forschungsgemeinschaft (grants KN 152/19-1,2) is gratefully acknowledged. The Vacuum Tower Telescope is operated by the Kiepenheuer-Institut für Sonnenphysik in Freiburg (Germany) in the Spanish Observatorio del Teide of the Instituto de Astrofísica de Canarias in Tenerife. J.H. thanks the Universitäts-Sternwarte Göttingen for the hospitality and the financial support.

\section{References}

Bendlin, C., \& Volkmer, R. 1995, A\&AS, 112, 371

Bendlin, C., Volkmer, R., \& Kneer, F. 1992, A\&A, 257, 817

Brault, J. W., \& Neckel, H. 1987, Spectral Atlas of Solar Absolute Disc-averaged and Disc-center Intensities from 3290 to $12510 \AA$, unpublished

Brault, J. W., \& White, R. O. 1971, A\&A, 13, 169

Carlier, A., Chauveau, F., Hugon, M., \& Rösch, J. 1968, Comp. Rend. Acad. Sci., 266, 119

Cattaneo, F., Brummell, N. H., Toomre, J., Malagoli, A., \& Hurlburt, N. E. 1991, ApJ, 370, 282

Collados, M., Rodríguez Hidalgo, I., Ballesteros, E., et al. 1996, A\&AS, 115, 367

de Boer, C. R. 1993, Ph.D. Thesis, Universität Göttingen

Deubner, F.-L. 1974, Solar Phys., 39, 31

Deubner, F.-L. 1988, A\&A, 204, 301

Espagnet, O., Muller, R., Roudier, T., Mein, N., \& Mein, P. 1995, A\&AS, 109, 79

Hirzberger, J., Vázquez, M., Bonet, J. A., Hanslmeier, A., \& Sobotka, M. 1997, ApJ, 480, 406

Hirzberger, J., Bonet, J. A., Vázquez, M., \& Hanslmeier, A. 1999a, ApJ, 515, 441

Hirzberger, J., Bonet, J. A., Vázquez, M., \& Hanslmeier, A. 1999b, ApJ, 527, 405

Holweger, H., \& Müller, E. 1974, Solar Phys., 39, 19

Johannesson, A., Bida, T., Lites, B., \& Scharmer, G. B. 1992, A\&A, 258, 572

Keller, C. U., \& von der Lühe, O. 1992, A\&A, 261, 321

Kitai, R., \& Kawaguchi, I. 1979, Solar Phys., 64, 3

Kneer, F., Mattig, W., Nesis, A., \& Werner, W. 1980, Solar Phys., 68, 31

Komm, R., Mattig, W., \& Nesis, A. 1991, A\&A, 252, 812

Koschinsky, M., Kneer, F., \& Hirzberger, J. 2001, A\&A, in press

Krieg, J., Wunnenberg, M., Kneer, F., Koschinsky, M., \& Ritter, C. 1999, A\&A, 343, 983

Krieg, J., Kneer, F., Koschinsky, M., \& Ritter, C. 2000, A\&A, 360,1157

Löfdahl, M. 1996, Ph.D. Thesis, Stockholm University

Mein, P. 1971, Solar Phys., 20, 3

Muller, R. 1985, Solar Phys., 100, 237

Muller, R. 1999, The Solar Granulation, in Motions in the Solar Atmosphere, ed. A. Hanslmeier, \& M. Messerotti (Kluwer, Dordrecht), 35

Nesis, A., Durrant, C. J., \& Mattig, W. 1988, A\&A, 201, 153

Nesis, A., Hanslmeier, A., Hammer, R., et al. 1992, A\&A, 253, 561

Nordlund, Å. 1985, Solar Phys., 100, 209

Nordlund, A., Spruit, H. C., Ludwig, H.-G., \& Trampedach, R. 1997, A\&A, 328, 229

Noyes, R. W., \& Leighton, R. B. 1963, ApJ, 138, 631

Rast, M. 1995, ApJ, 443, 863

Roudier, T., \& Muller, R. 1987, Solar Phys., 107, 11

Salucci, G., Bertello, L., Cavallini, F., Ceppatelli, G., \& Righini, A. 1994, A\&A, 285, 322

Spruit, H. C., Nordlund, A., \& Title, A. M. 1990, ARA\&A, 28, 263

Steffen, M., Ludwig, H. G., \& Krüß, A. 1989, A\&A, 213, 371

Stein, R. F., \& Nordlund, Å. 1998, ApJ, 499, 914

Stix, M. 1989, The Sun, An Introduction (Springer, Berlin), $263 \mathrm{ff}$

Title, A. M., Tarbell, T. D., Topka, K. P., Ferguson, S. H., \& Shine, R. A., the SOUP Team, 1989, ApJ, 336, 475

von der Lühe, O. 1984, J. Opt. Soc. Am., A1, 510

Weigelt, G. P. 1977, Opt. Comm., 21, 55 